\title{
Elementos externos e internos en un cambio de enfoque etnográfico. El caso de una investigación sobre antropología política
}

A change of approach in ethnography and its external and internal components. The case of a research on political anthropology

\author{
Luca Sebastiani \\ Investigador pre-doctoral del Departamento de Antropología Social y el Instituto de Migraciones, Universidad de Granada. España \\ lucaseba78@ugr.es
}

\begin{abstract}
RESUMEN
La actividad investigadora, lejos de ser un proceso lineal, está a menudo caracterizada por elementos de incertidumbre, "vueltas atrás" y avances repentinos, tanto en las ciencias sociales como en las naturales. Partiendo de este supuesto, en el presente artículo ilustraré el caso de mi tesis doctoral, que es un análisis crítico de las políticas de integración de inmigrantes de la Unión Europea. Recorreré las principales transformaciones sufridas por el objeto y el contexto de estudio y analizaré los diferentes factores que han influido en el procedimiento investigador, tanto "internos" como "externos" a su lógica de desarrollo. Asimismo, dialogaré con algunas fuentes teórico-metodológicas que me han resultado particularmente útiles para pensar el objeto y el contexto de estudio desde una perspectiva compleja y relacional. En este sentido, proporcionaré las principales contribuciones aportadas por la antropología de las políticas públicas, los estudios sobre gubernamentalidad y la etnografía "no local".
\end{abstract}

ABSTRAC T

Scientific research is not a linear process; rather it is often characterized by uncertainties, "backtrackings" as well as sudden advances, both in social and natural sciences. Starting from this assumption, in this paper I will analyse the specific case of my $\mathrm{PhD}$ thesis, which is a critical analysis of the European Union's policies on immigrant integration. I will show the main transformations undergone by the object and the context of study, focusing on the different factors (both "internal" and "external") that have influenced my research. . Also I will focus on some theoretical-methodological contributions that have been particularly useful in order to examine the object and the context of study from a complex and relational perspective. In this sense, I will show the main contributions given by the anthropology of policy, governmentality studies, and "non-local" ethnography.

PALABRAS CLAVE

objeto de estudio | contexto de estudio | etnografía "no local" | antropología de las políticas públicas | gubernamentalidad | dispositivo KEYW O RDS

object of study | context of study | "non-local" ethnography | anthropology of policy | governmentality | apparatus

\section{Introducción}

Que la actividad investigadora suela transitar por andares tortuosos y (a veces) hasta inciertos es un hecho aceptado por la comunidad científica. En efecto, el acercamiento a los fenómenos estudiados no se despliega como un encadenamiento lineal de etapas previamente definidas: formulación de interrogantes, ubicación epistemológica, definición del marco teórico-metodológico y elección de las técnicas oportunas. Los planteamientos que lleguemos a considerar adecuados para nuestra estrategia investigadora raras veces son la desembocadura lógica de las anteriores elaboraciones, ni simplemente representan una descripción aséptica de la realidad. Después de las aportaciones de autores como Kuhn (1975), Hesse (1980), Feyerabend (1987), Morin (2004), etc., incluso en las ciencias naturales hoy en dia es asumido que numerosos factores de diversos tipos inciden en el desarrollo del proceso de investigación. En el caso de las ciencias sociales, aún más cabe subrayar tanto la existencia de elementos "internos" a la lógica de desarrollo de un trabajo, como de circunstancias "externas" -ya se trate de condicionantes socioculturales, económicos o hasta vivenciales-. Estas influencias pueden originar de encuentros significativos, vicisitudes o disyuntivas; en todo caso, se encuentran imbricadas en las experiencias que atravesamos. Aluden a nuestra condición de sujetos que hablan desde una geo-política y corpo-política del conocimiento (Mignolo 2009), producen saberes situados (Haraway 1995) y encarnan unos habitus (Bourdieu y Wacquant 2005). Asimismo, se enmarcan dentro de ciertas condiciones de producción históricas. Suelen llegar de manera intempestiva, casi nunca a tiempo, y a veces remiten a la creatividad, el azar o la intuición antes que a la 
causalidad.

Asumiendo este marco epistemológico como punto de partida, en el presente artículo analizaré la experiencia de mi tesis doctoral (1), un trabajo enfocado al análisis de las políticas públicas contemporáneas desde una perspectiva transdisciplinar, en el cruce entre antropología y sociología cualitativa. Entendiendo esta investigación como un ejemplo más de algunas cuestiones anteriormente esbozadas, ilustraré su recorrido zigzagueante mediante una discusión sobre las principales modificaciones sufridas por el objeto y el contexto de estudio a lo largo del camino. Al hacerlo, también llamaré la atención sobre algunos de los condicionantes "internos" y "externos" que han influido en el avance del procedimiento en su conjunto. Además, desarrollaré reflexiones teórico-metodológicas que considero particularmente novedosas en mi ámbito de especialización, estableciendo un diálogo con las fuentes que más me han ayudado a afrontar las dificultades encontradas. Si bien toda investigación tiene sus peculiaridades, estimo que algunas de las propuestas aquí esbozadas pueden proporcionar útiles sugerencias para otros trabajos que aborden problemáticas parecidas a la mía.

La estructura del artículo es la siguiente: primero esbozaré mi formación previa a la elaboración del diseño de tesis doctoral, las inquietudes y los interrogantes, para mostrar de qué manera el objeto de estudio inicial ha ido transformándose hasta llegar a su formato definitivo. Igualmente, rescataré algunas claves de reflexión teórica relacionadas con la antropología de las políticas públicas y los estudios sobre gubernamentalidad. Luego me centraré en el contexto, comentaré sus modificaciones y argumentaré la necesidad de concebirlo como un espacio relacional antes que un lugar geográfico. A continuación, comentaré la relevancia del concepto "dispositivo" en tanto que herramienta central para el análisis de los ámbitos multisituados e interconectados de producción de las políticas públicas actuales. Finalmente, ilustraré las implicaciones que esto conlleva para la práctica etnográfica y recapitularé las principales conclusiones.

\section{Y sin embargo se mueve: los desplazamientos del objeto de estudio}

\subsection{Los primeros acercamientos}

Me licencié en Ciencias Políticas por la Universidad de Bolonia (2006). Mi trabajo de licenciatura era un análisis comparativo de los modelos de integración implementados en cuatro países europeos ante los inmigrantes procedentes de países "musulmanes". Su enfoque era básicamente prescriptivo, ya que estaba orientado a determinar las medidas más eficaces y no presentaba inquietudes epistemológicas: de hecho, nociones como "multiculturalismo" e "integración" eran asumidas de manera relativamente aproblemática.

En 2008 me matriculé en el Doctorado en Antropología Social y Diversidad Cultural de la Universidad de Granada, presentando un diseño de tesis cuyo título recitaba: "Concepción de la identidad europea e inmigración: un análisis de los discursos dominantes entre los políticos de la Unión Europea". Asumiendo que la inmigración es un "hecho político radical" (De Lucas 2003) y el estudio de las políticas migratorias constituye una herramienta de "sociología del estado" (Sayad 2002), consideraba que las políticas de integración, al centrarse en las condiciones exigidas a determinados colectivos de extranjeros para ser considerados miembros a pleno título de la sociedad receptora, nos hablan indirectamente de la idea de pertenencia manejada por quienes las elaboran (véase Favell 2000). Si bien algunas investigaciones se habían cimentado en este enfoque para analizar procesos de construcción nacional (véase Gil 2006), yo quería aplicarlo al nivel de la UE, poniendo en relación los discursos de los parlamentarios europeos con la (posible) existencia de una dinámica de producción de una identidad "supranacional" y "comunitaria". El concepto de integración, entendido en el trabajo de licenciatura como una realidad objetiva, se había convertido en un instrumento útil para detectar otros procesos sociales.

El título del trabajo presentado para la obtención del Diploma de Estudios Avanzados (2010) era "Inmigración e integración de inmigrantes: políticas comunitarias y proceso de construcción identitaria de la UE". La noción de identidad seguía ocupando un espacio relevante, si bien era crecientemente problematizada -siendo definida como una noción "relacional" y "procesual" antes que "esencial y "estática" (Bhabha 2003, Bauman 2003 y Hall 2003)- . Además, otras perspectivas integraban el marco teórico de este trabajo: la antropología de las políticas públicas y los estudios sobre gubernamentalidad. 
El primer acercamiento, en lugar de asumir como dadas las clasificaciones y categorías de las políticas públicas, las considera problematizaciones a deconstruir; por tanto, se centra en el análisis crítico de las racionalidades y los supuestos socio-culturales subyacentes en aquéllas (Shore y Wright 1997). Desde esta perspectiva, se considera que el objetivo de las políticas públicas no es el de formular soluciones racionales ante los problemas "realmente" existentes para conseguir resultados. Más bien, éstas tienen un carácter performativo y contribuyen, mediante las prácticas (discursivas y no discursivas) que vertebran, a (re)presentar y (re)producir la realidad de una manera determinada; a abrir ciertos horizontes de posibilidades y cerrar otros; a consolidar (o subvertir) desigualdades, procesos de inclusión/exclusión y relaciones de fuerzas asimétricas (Shore y Wright 1997, y Shore y otros 2011). Analizar críticamente las políticas públicas conlleva no limitarse a estudiar los "objetos" nombrados por aquéllas ni tampoco a los destinatarios de sus medidas, sino sobre todo dirigirse a los mecanismos de producción de clasificaciones y subjetividades; una actitud que ha sido eficazmente definida "ponerse el ojo en la espalda" (Agrela 2006: 18). Es que las políticas públicas, a pesar de ser presentadas de manera "científica", confeccionadas en la aparente neutralidad del "saber experto", son producidas por actores situados (Shore y Wright 1997). Lo cual no quiere decir que exista un modelo de implementación jerárquico y unidireccional, sino más bien que éstas se encuentran imbricadas en una red de actores, saberes, mecanismos variados y a veces adquieren una agencia y una "vida propia" y producen resultados inesperados, más allá de las intenciones de sus creadores (Shore 2010).

Los estudios sobre gubernamentalidad originan de la reflexión foucaultiana tardía y de los sucesivos desarrollos de algunos seguidores del filósofo francés (véanse Burchell y otros 1991, Rose 1999 y Dean 2010). Desde esta perspectiva, el gobierno es definido como la "conducción de la conducta de uno mismo y de los demás" (Foucault 1991: 88) y alude al hecho de que el ejercicio del poder, al menos a partir del siglo XVIII, no se da exclusivamente a través del dominio o la coerción, sino mediante la implicación de los individuos y grupos gobernados, los cuales, asumiendo en sí mismos los objetivos gubernamentales, participan activamente en su "libre" implementación. Este concepto es útil para ilustrar la profunda vinculación existente entre relaciones de poder y procesos de subjetivación. Otra noción es la de gubernamentalidad, que remite al "cómo" del gobierno (Foucault 1991: 102-103), a las formas concretas de gobernar que deben ser utilizadas en un contexto dado. Finalmente, la expresión "racionalidades políticas" indica las formas de conceptualizar el ejercicio del poder, incluyendo las justificaciones relativas al objeto, los límites de la acción política y su reparto entre diferentes autoridades (Rose y Miller 1992: 3). ¿Qué quería decir para mi tesis adoptar este enfoque? Otros estudiosos ya lo habían aplicado en el ámbito de las políticas nacionales de integración de inmigrantes, utilizando estas últimas como una herramienta para detectar procesos sociales más amplios (véase Gil 2006). En la misma línea, aunque en un plano diferente (el comunitario), me preguntaba: ¿por qué se teoriza la integración de inmigrantes como algo necesario sólo a partir de cierto momento histórico?; ¿por qué las medidas de integración se dirigen sólo a ciertos colectivos de inmigrantes?; ¿qué tecnologías se están empleando para implementarlas y con qué objetivos? En definitiva: ¿qué es lo que nos indica el gobierno de ciertas poblaciones desde la perspectiva de la integración? Al tratar de responder, resaltaba que, en el contexto de lo que ha sido llamado "metamorfosis de la cuestión social" (Castel 1997) o "racionalidad política neoliberal" (Rose y Miller 1992: 30-34), el creciente repliegue de las identidades laborales colectivas ha sido reemplazado por el auge de los discursos "culturalistas" y "étnicos" sobre la pertenencia. Lo cual conlleva otras formas de teorizar y gestionar la diversidad desde los poderes públicos.

Como puede verse, la perspectiva analítica de mi futura tesis iba haciéndose más compleja. Aun así, todavía consideraba que ésta tendría un objeto de estudio dúplice: por un lado el proceso identitario europeo y por el otro el análisis crítico de las políticas públicas desde el enfoque de la gubernamentalidad.

\subsection{El abandono de las temáticas identitarias}

Conforme avanzaba, comprendí que la "identidad europea" como objeto de estudio entrañaba una serie de incertidumbres y complicaciones. Entre otras: ¿por qué tener en cuenta solamente los discursos de los parlamentarios europeos?; ¿de esta manera, no estaba proponiendo una idea formalista e institucional de la práctica política?; ¿no sería oportuno tener en cuenta también otros procesos identitarios, relativos a espacios más "profanos"? Por otra parte: ¿qué era la "identidad europea"? Desde la perspectiva de la gubernamentalidad, Walters y Haahr analizan la Unión Europea como un intento de "gubernamentalización 
de Europa", mediante su transformación en un "objeto conocible y gobernable como un espacio de procesos sociales, económicos y políticos" (Walters y Haahr 2005: 10). En cambio, mi diseño original, al asumir casi naturalmente la correspondencia entre los conceptos de "Europa" y "Unión Europea", ¿no estaba naturalizando una categoría (potencialmente) esencialista en lugar de problematizarla?

Si bien estas objeciones contribuyeron al inminente abandono de la pregunta identitaria, ninguna fue el detonante principal, ya que la experiencia "terrenal" del trabajo de campo me empujó definitivamente a revisar la formulación de ciertos interrogantes. En efecto, al volver de mi primera estancia en Bruselas (febrero/marzo de 2011) y tras realizar una tanda de 18 entrevistas, vi que las conversaciones no aportaban materiales particularmente útiles sobre la cuestión identitaria. En cambio, había contribuciones muy interesantes relativas al eje de la gubernamentalidad. Ante este impedimento práctico decidí renunciar al primer tema de investigación para centrarme en el segundo: de hecho, en las entrevistas sucesivas el eje de interrogantes sobre la identidad sería abandonado.

A lo largo de un proceso difícil de resumir en pocas líneas -compuesto por otra estancia en Bruselas (junio de 2012), dos viajes puntuales de pocos días, observaciones de varios tipos, participaciones en congresos y seminarios, conversaciones con personal investigador, etc.- llegué a una aproximación satisfactoria al objeto de estudio, en la que las cuestiones teóricas estaban formuladas de una manera definitiva que voy a exponer. Mi actual tesis se basa en la perspectiva de la gubernamentalidad y en la deconstrucción de las políticas comunitarias de integración de inmigrantes. En ella realizo un estudio de las prácticas y los instrumentos implementados dentro de este marco político, mostrando que éstos, lejos de constituir medidas "neutrales" y "eficaces" orientadas a regular las interacciones entre "autóctonos" e "inmigrantes", están imbuidos de supuestos socioculturales y se encuentran imbricados en relaciones de fuerzas asimétricas. Destaco en particular los siguientes aspectos:

\section{1) El concepto de integración es una construcción social.}

2) Esta noción se ha ido definiendo mediante un proceso de (aparente) despolitización y tecnificación, donde el debate sobre las mejores prácticas a implementar ha tenido en cuenta las soluciones, "basadas en la evidencia" y definidas "realistas", disponibles dentro de un repertorio limitado. Sin embargo, en ningún momento se ha cuestionado el contexto general de desigualdades estructurales en el que ciertas políticas acontecen (más bien éstas han sido asumidas y naturalizadas).

3) Este entendimiento de la integración ha legitimado y (a veces) impulsado procesos de inclusión/exclusión; entre otras cosas, invisibilizando ciertas experiencias contrahegemónicas de luchas migrantes o poniendo al margen las vivencias de quiénes no estén reconocidos por el marco interpretativo establecido; por ejemplo, las personas migrantes indocumentadas, excluidas de las políticas de integración comunitarias a raíz de su condición administrativa.

4) La Unión Europea ha fomentado unos patrones específicos de participación de la sociedad civil en los mecanismos de elaboración/implementación/evaluación de las políticas de integración, en los que la agencia de aquélla es atravesada por un intento de "captura" y "encauzamiento" dentro de los objetivos gubernamentales.

La formulación final evidencia con claridad la relevancia de las transformaciones sufridas por el objeto de estudio, el "qué" de mi tesis. Lo que había nacido como un estudio "racional" de las políticas públicas se fue alejando de las influencias politológicas, para convertirse finalmente en un análisis crítico de las relaciones de poder/saber (sobre este concepto véase Foucault 2009) hegemónicas y su orden de verdad. En este contexto, la misma noción de integración había pasado de ser un objeto de estudio aproblemático a convertirse en un pretexto para analizar procesos políticos y sociales de más amplio calado.

Naturalmente, si hubiera tenido claro desde el principio cómo enfocar la investigación, los guiones de las primeras entrevistas habrían sido diferentes; sin embargo, al no poder volver atrás, tuve que re-enfocar la perspectiva y realizar ajustes sobre la marcha. Lo mismo pasaba con los planteamientos teóricos: las nuevas reflexiones, no necesariamente incompatibles con las anteriores, a veces se superponían a aquéllas; otras terminaban reemplazándolas; en ocasiones se sedimentaban varias "tiras" argumentativas, originarias de diferentes fases de la investigación. En este proceso no se ha dado ningún determinismo lógico, puesto que el objeto de estudio ha estado condicionado por diferentes vicisitudes, tanto de tipo teórico como vital. En particular, los tiempos de realización del trabajo de campo se han visto afectados por los 
constreñimientos económicos a los que remitía mi condición (entonces) de investigador pre-doctoral en formación, financiado por una beca y sucesivamente un contrato FPU.

\section{Los desplazamientos del contexto}

\subsection{Acotando los límites geográficos: Bruselas como campo}

Volvamos ahora atrás, al comienzo de mi investigación, para recorrer las vicisitudes del contexto de estudio, el "dónde" (2) de mi tesis. En la formulación del primer diseño había definido los "límites geográficos" de la siguiente manera: teniendo en cuenta que la relación entre electorado y representantes políticos se da principalmente en el plano estatal (incluso en los comicios europeos), planeaba centrarme en los contextos español, italiano, holandés y rumano. Para los futuros cuatro años tenía planificadas cinco estancias diferentes: una "central" en las dos sedes del Parlamento Europeo (Estrasburgo y Bruselas) para entrevistar a sus miembros y otra para cada país. En los ámbitos estatales me encargaría de observar acontecimientos vinculados a las políticas de integración y a su relación con la construcción de la identidad europea -mítines electorales, actuaciones institucionales, casos mediáticos, etc.-. El marco politológico aún central emergía de las preocupaciones por el carácter extensivo del trabajo y desembocaba en una pretensión comparativa exageradamente ancha.

Mientras tanto mi formación antropológica iba ampliándose, entre otras cosas, gracias a la actividad docente que desempeñaría en los dos años posteriores en la asignatura de Antropología Social, impartida respectivamente en el segundo grado de Trabajo Social (A.A. 2011/2012) y el quinto año de licenciatura en Sociología (2012/2013) de la Universidad de Granada. Conforme incorporaba los requerimientos "clásicos" del trabajo de campo, entendí que era necesario reducir la amplitud geográfica del contexto, especialmente en dos sentidos: 1) disminuyendo el número de ámbitos nacionales de referencia; 2 ) identificando, dentro de los mencionados contextos, micro-unidades locales en las que aterrizar la labor etnográfica. Esta idea se plasmó en la formulación del trabajo para la obtención del DEA, que reducía los contextos estatales a dos: el español y el italiano. Además, había empezado a revisar diariamente las ediciones online de dos periódicos italianos (La Repubblica e II Corriere), dos españoles (El País y El Mundo) y un boletín de noticias de la UE (Euronews), con la finalidad de guardar los artículos más interesantes relacionados con la temática de investigación. Tenía pensado seleccionar posteriormente algunos eventos, acontecidos en los países escogidos o en el ámbito institucional de la Unión Europea, que pudieran constituir un argumento de discusión con los futuros entrevistados o me indicaran contextos específicos de observación a los que dirigirme. En este sentido, la idea de abarcar diferentes contextos nacionales empezaba a emanciparse del afán comparativo por detectar "analogías" y "diferencias", para en cambio centrarse en el análisis de las maneras en que el mismo objeto de estudio -a esas alturas en realidad eran dos: la "identidad europea" y el estudio de las gubernamentalidades- estaba siendo resignificado en situaciones diferentes. Bajo estos supuestos metodológicos, a principios de 2011 me dirigí al contexto "europeo" de Bruselas y "empecé" el trabajo de campo, con la idea (nunca hecha realidad) de moverme sucesivamente a Estrasburgo y a los contextos "locales-nacionales", que también estaban en fase de definición.

\subsection{La idea de campo se transforma: de lugar geográfico a espacio relacional}

Al llegar a Bruselas establecí los primeros contactos, empecé a concertar las futuras entrevistas y también realicé algunas observaciones preliminares del "barrio europeo", en el que tienen sede las principales instituciones de la UE, así como numerosos grupos de presión y ONG. Tomando como referencia otros trabajos etnográficos (véanse Abélès 2000 y 2009, Abélès y otros 1993, Bellier 1997 y 2000, Bellier y Wilson 2000, Shore 1993, 2000 y 2001 y Shore y Black 1996), pensaba que la observación de los lugares, los edificios, las personas, etc., me ayudaría a aprehender los mundos sociales habitados por los "profesionales de Europa" (Abélès 2009). Además, esta práctica sonaba muy antropológica, ya que remitía a una representación tradicional de la labor etnográfica. No obstante, rápidamente me di cuenta de que la observación no aportaba ulteriores informaciones a las que ya sabía, ni tampoco lograba encontrar un nexo estrecho entre éstas y el objeto de estudio de mi tesis.

A todo esto se añadió otra dificultad: el gran desgaste en términos logísticos y de tiempo -y por consiguiente, también financieros- a la hora de contactar con los grupos parlamentarios. En efecto, para conseguir una entrevista con algún representante era necesario: 1) detectar a aquéllos que estuvieran más informados sobre las políticas de integración, por lo general, miembros de la Comisión "Libertades Civiles, Justicia y Asuntos de Interior" (LIBE); 2) que éstos contestaran a mis correos electrónicos de presentación; 
3) pasar el filtro de una conversación con los secretarios particulares; 4) finalmente, concertar una cita con los parlamentarios, siempre y cuando tuvieran el tiempo y las ganas de hacerme un hueco en su ajetreada agenda. Por supuesto, se trataba de dificultades inherentes al trabajo de campo y con las que lidiar, si no me hubiera dado cuenta de que el Parlamento Europeo no era un actor tan relevante como pensaba. De hecho, ocupaba un espacio secundario en las políticas comunitarias de integración, mientras que otros actores llevaban la voz cantante en este ámbito (3). Esta falta de protagonismo se debe, entre otras cosas, a que la Unión Europea (art. 79.4 del Tratado de Lisboa) no tiene la facultad de legislar en esta esfera y sólo puede establecer medidas de apoyo a las políticas nacionales. Es éste un dominio de "políticas blandas" (soft law), donde la implementación de medidas comunitarias tiene que basarse en su aceptación voluntaria por parte de los estados miembros (4).

Después de mejorar mi conocimiento sobre los mencionados mecanismos, me di cuenta de que la institución europea más implicada en las negociaciones sobre las políticas de integración era la Comisión, aunque no tuviera ningún instrumento vinculante para hacer respetar las decisiones tomadas. Además, conforme iba ampliando los contactos comprendí que al lado de la Comisión existía una gran variedad de actores activos en la elaboración/implementación/evaluación de las diferentes medidas, no todos estrictamente institucionales. Podía tratarse de funcionarios clave del Comité Económico y Social, del Comité de las Regiones o también de representantes de gobiernos nacionales o administraciones locales; igualmente, había multitud de exponentes de la sociedad civil, ya fueran ONG "paraguas" -con sede en Bruselas e integradas por organizaciones estatales-, expertos y académicos, miembros de think-tank o de fundaciones europeas. Este descubrimiento me llevó a relativizar el papel de los parlamentarios y me empujó a dirigirme a ese variado abanico de actores, no sólo más implicado en mi temática de estudio, sino también (afortunadamente) más fácil de contactar de cara a una entrevista. A esas alturas entendí que no tenía sentido insistir, ni en la idea de entrevistar a miembros del Parlamento, ni en la determinación de realizar observaciones en otros contextos locales. De manera consecuente, interrumpí la búsqueda sistemática de noticias en los periódicos nacionales, que me había supuesto un gran desgaste de tiempo. Por fin había asumido plenamente, gracias a la experiencia práctica, la necesidad de realizar un estudio intensivo y sin pretensiones comparativas.

Conforme realizaba las primeras entrevistas, también fui profundizando el conocimiento de los instrumentos predispuestos por la UE en materia de integración. En particular me centré en el análisis del "Marco Europeo de la Integración", expresión que remite al conjunto de las políticas blandas desarrolladas en los últimos 15 años, con el objetivo de fomentar un entendimiento común de esta noción entre los actores anteriormente mencionados y establecer un espacio de debate. De manera extremadamente resumida, mencionaré sus instrumentos más importantes:

1) Once "Principios Básicos Comunes" (2004), aprobados por el Consejo de Justicia y Asuntos Interiores y enfocados a dar una definición oficial de la integración y de sus principales componentes.

2) Tres ediciones del "Manual sobre la Integración para responsables de políticas y profesionales" (2004, 2007, 2010), repletos de recomendaciones y buenas prácticas a difundir.

3) Una plataforma participativa llamada "Foro Europeo sobre la Integración" (2009).

4) Herramientas virtuales como el "Sitio Web Europeo sobre la Integración” (2009).

5) El "Fondo Europeo para la Integración" (2007-2013), destinado a apoyar proyectos transnacionales y nacionales orientados a la implementación de los Principios Básicos Comunes.

6) Debates sobre la construcción de indicadores para evaluar las políticas nacionales y "módulos" para operacionalizar ulteriormente el intercambio de buenas prácticas (a partir de 2010).

Ante este escenario, mi idea era la de acercarme al conjunto de los anteriores instrumentos, para en un segundo momento elegir esmeradamente uno, en el que asentar un estudio de caso. Una vez asumido el enfoque "intensivo", no me parecía viable abordar todas las herramientas existentes. Además, cada una parecía tener su propia lógica de funcionamiento, remitiendo a situaciones muy diferentes y poco homogéneas: no quería proponer una efímera "suma" de temáticas sin hilo común. En este sentido, me llamó la atención el "Foro Europeo sobre la Integración", una plataforma patrocinada por la Comisión Europea y el Comité Económico y Social que se reúne en Bruselas dos veces al año e integra alrededor de cien 
organizaciones de la sociedad civil (europea y nacional), además de representantes institucionales de todos los niveles y exponentes del saber experto. Paulatinamente, maduré la idea de convertir el Foro en mi contexto de investigación, planteándome dos objetivos: 1) analizar las diferentes resignificaciones producidas por sus miembros sobre el concepto de integración; 2) indagar sobre las prácticas participativas desarrolladas por la sociedad civil en ese ámbito. El proyecto se llevaría a cabo mediante entrevistas a sus protagonistas (ya en fase de realización) y observaciones directas de sus sesiones.

Sin embargo, otra vez más, me di cuenta de que "algo no funcionaba". Estaba persistiendo en un error: el de querer acotar las unidades de análisis mediante una reducción de las dimensiones del campo, reemplazando la noción algo difuminada de "Bruselas" por un contexto más limitado y abarcable, sin entender que el problema mayor radicaba en la concepción geográfica del contexto de estudio. A esta dificultad me remitía el propio procedimiento investigador: en efecto, por mucho que el Foro se reuniera en un lugar determinado (marcadamente, los locales del Comité Económico y Social), éste en absoluto agotaba el entramado de relaciones tejido por sus miembros, ni abarcaba la totalidad de los universos socio-culturales relevantes para aquéllos. En otras palabras, lo que ocurría dentro del Foro no podía entenderse sin tener en cuenta lo que acontecía fuera de él (sobre esta idea véanse Marcus 1995: 111 y García y otros 2011: 218), ya que, por poner un ejemplo, para algunos miembros de las instituciones y la sociedad civil "europeas" esta plataforma constituía sólo una de las numerosas ocasiones de encuentro; sólo representaba uno de los momentos en los que se compartían y producían lenguajes, problematizaciones y maneras de ver las cosas. Por otra parte, para muchos representantes de nivel estatal -especialmente las ONG nacionales- Bruselas constituía poco más que un lugar al que las organizaciones viajaban dos veces al año y el Foro no representaba para nada una prioridad de la acción política cotidiana. Por tanto, la experiencia socio-cultural de los actores presentes en el Foro y la producción de prácticas políticas relevantes para aquéllos no se reducían bajo ningún concepto al contexto bruselense, ni tampoco a las dinámicas internas de la plataforma. En este sentido, el Foro no podía ser conceptualizado como un "campo" por sí mismo, geográficamente separado de otros campos y en el que se produjeran procesos "aislados" relacionados con las políticas de integración. No representaba ninguna totalidad a "contener" y aprehender de una manera holística mediante observaciones intensivas y entrevistas. Además, las prácticas desarrolladas por los actores activos en esta plataforma me invitaban claramente a cuestionar una representación simplista de sus propios planos de interacción. Formularé un ejemplo para aclarar el concepto: dentro del Foro es posible observar una alianza discursiva entre la Comisión, algunas ONG europeas y nacionales así como ciertas redes de administraciones locales, enfrentadas a otros actores "restrictivos", en la mayoría de los casos gobiernos nacionales. En este contexto, hablar de políticas comunitarias no quería decir entenderlas como un nivel "superior" a los anteriores acorde con una representación piramidal de los procesos decisionales, ni tampoco remitir a los actores formalmente "supranacionales". Por el contrario: abarcar la dinámica en la que se constituían las políticas públicas comunitarias y se producía su sentido implicaba moverse a través de diferentes planos.

En definitiva: el error consistía en concebir el contexto de estudio como un "dónde" geográfico (o como una suma de "dóndes"). Por el contrario, de lo que se trataba era entenderlo como un conjunto de relaciones, producidas en diferentes niveles a través de interacciones significativas entre los actores. Así pues, entendí que no había que ir a ningún "centro" geográfico, más bien había que moverse en las intersecciones entre diferentes espacios. Mediante la experiencia empírica llegué a conceptualizar el campo de una manera bastante diferente a la inicial: ya entendía que el "dónde" no era una simple noción geográfica -un lugar-, sino un espacio atravesado por interconexiones, una articulación compleja de prácticas, un entrecruce de actores y niveles diferentes, un contexto compuesto por multiples contextos. Mi tarea, entonces, no sería la de estudiar un único lugar (ya fuera "Bruselas" o el Foro), ni tampoco más lugares (tal y como me había propuesto con la idea de las observaciones locales), sino de estudiar en/con lugares (García y otros 2011: 205) o, mejor aún, a través de lugares (Reinhold 1994 en Shore y Wright 1997: 14) (5). Adoptar el planteamento arriba indicado conllevaba cuestionar ciertas categorías tradicionales -"micro", "macro", "local", "nacional", "supranacional", etc.- tan arraigadas en lo que ha sido llamado "nacionalismo metodológico" (véanse Wimmer y Schiller 2002 y Favell 2006) las cuales, debido a su lógica jerárquica unidireccional, no siempre resultaban adecuadas para desentrañar la complejidad de las relaciones a analizar. En lugar de solapar el contexto de estudio a las realidades sociales pre-existentes (tal y como había hecho con respecto del Foro), entendí que debía "allanar" el mundo social y dejarme guiar por las formas en las que los propios actores iban "formateándolo", organizando significados y clasificaciones (Latour 2005: 227). Se trataba entonces de desarticular la lógica jerárquica arriba mencionada y adoptar una perspectiva transescalar (García y otros 2011). Sólo después de esta operación sería posible 
"reconectar" los espacios y establecer escalas adecuadas para representar las relaciones relevantes en mi contexto específico (Latour 2005: 220).

Analizando mi procedimiento de investigación desde el conocimiento actual, cabe preguntarse si la razón inicial por la que me dirigí a Bruselas fue que esta ciudad, al ser considerada la "capital de Europa", me permitía proporcionar un "centro" a esa entidad tan "evanescente" llamada Unión Europea. De esa manera estaba adoptando una representación tradicional del espacio geográfico y sus ordenaciones jerárquicas, la cual sin embargo no se correspondía a la fragmentada articulación de las relaciones relevantes en la producción de las políticas públicas sobre la integración. Mi necesidad original de establecer un campo "palpable", tangible y material, aun sonando tranquilizadora me estaba conduciendo al error de inscribir el conjunto de relaciones analizadas dentro de un territorio geográfico, a pesar de que estas últimas no se manifestaban en un espacio meramente físico. En este sentido hay que señalar que, aunque finalmente mantuve la decisión de situarme en Bruselas -es más, la ciudad ha representado mi posicionamiento geográfico principal-, esta determinación llegó a adquirir otro sentido. Ya no constituía el lugar "por excelencia" en el que estaban contenidos los fenómenos socio-culturales a observar, sino más bien representaba una localización (Gupta y Ferguson 1997, Feldman 2011), indudablemente privilegiada pero tampoco única, útil para analizar procesos políticos más amplios (Wright 2011: 27). Aterrizar la investigación en un lugar concreto representaba un posicionamiento estratégico desde el que construir conexiones (Marcus 1995: 99) y enlaces epistemológicos y políticos (Gupta y Ferguson 1997: 5) con otros espacios, no con la ambición de cubrirlos todos, sino de aprehender las relaciones relevantes, las cuales se encontraban inscritas en fenómenos socio-culturales multisituados (García y otros 2011: 206).

\subsection{Re-construyendo el campo mediante el concepto de dispositivo}

En su propuesta de etnografía multisituada, Marcus aboga por una estrategia orientada a seguir personas, cosas, metáforas, tramas/historias/alegorías, vidas/biografías o conflictos (1995: 106-110). A ese listado, Shore y Wright añaden la posibilidad de "seguir a las políticas públicas" (2011: 12), recorrer su entramado relacional para agarrar los contornos del campo, que inevitablemente terminarán "expandiéndose" o "contrayéndose" según los casos (Wright 2011: 28) y asumirán una configuración definitiva bastante diferente de la pensada en un comienzo. Esto es lo que había estado haciendo yo, a pesar de no saberlo desde el principio. Es necesario insistir en que la elaboración explícita de mi enfoque metodológico fue un proceso "intempestivo" y "tardío", que se desenvolvió en estrecha conexión con las crecientes aclaraciones sobre el objeto de estudio. Incluso durante la segunda estancia en Bruselas (junio de 2012), en la que realicé otras 16 entrevistas, aún no había sido capaz de poner "punto y final" a la definición del contexto. Las entrevistas (abiertas y semi-dirigidas) realizadas proporcionaban una gran cantidad de materiales sobre diferentes cuestiones, ya que los actores, implicados (aunque en diferente medida) en la pluralidad de instrumentos comunitarios relacionados con la integración, me habían hablado de cosas como su experiencia en el Foro, el debate sobre los indicadores y los módulos, su relación con los otros protagonistas, el contexto socio-político general, los diferentes mecanismos participativos y un largo etcétera. La interconexión existente entre los actores, sus prácticas y la implementación de políticas se hacía a cada paso más evidente. De hecho, en las comunicaciones que había empezado a escribir para algunos congresos me ocupaba un poco de todos los diferentes instrumentos, lo cual resultaba bastante fácil ya que los materiales abundaban. De manera intuitiva, después de desechar la idea inicial de limitarme al Foro había vuelto a "expandir" el contexto de estudio, aunque desde un enfoque diferente. Sin embargo, percibía que aún me faltaba "algo": una fundamentación del campo más sólida, que le diera más coherencia y homogeneidad teórica.

Para eso resultó crucial la estancia en Bolonia en el verano de 2012. Allí tuve el tiempo de profundizar aún más en la bibliografía, hasta encontrar algunas lecturas muy útiles que finalmente me permitieron "nombrar" la práctica investigadora que había estado realizando hasta aquel entonces y hallar un un concepto clave para re-ordenar las coordenadas de mi quehacer etnográfico: se trata de la noción de "dispositivo". Foucault lo define así: "Un conjunto decididamente heterogéneo, que comprende discursos, instituciones, instalaciones arquitectónicas, decisiones reglamentarias, leyes, medidas administrativas, enunciados científicos, proposiciones filosóficas, morales, filantrópicas; en resumen: los elementos del dispositivo pertenecen tanto a lo dicho como a lo no dicho. El dispositivo es la red que puede establecerse entre estos elementos" (Foucault 1985: 128). Según Feldman, el esquema interpretativo proporcionado por esta noción apunta a un ámbito de estudio etnográfico crucial en la actualidad: aquél constituido por "regímenes de políticas públicas amorfos y descentralizados que funcionan regulando a grandes poblaciones" y las 
convierten en objetos cuantificables y gestionables (Feldman 2011: 32). En su propuesta de antropología de las políticas públicas, Shore y Wright destacan la necesidad de estudiar estos "ensamblajes entre agentes, instituciones, tecnologías y discursos" que funcionan como mecanismos de alineación entre los objetivos gubernamentales y la conducta de los actores (2011: 11). También añaden que esta noción, al poner en relación un conjunto de realidades diferentes (discursivas y no discursivas, humanas y no humanas), nos ayuda a teorizar la agencia a veces impersonal de las políticas públicas y sus consecuencias a menudo inesperadas (véanse Cruikshank 1999, Latour 2005, Shore 2010). Desde su perspectiva de "antropología de lo actual", Rabinow subraya que el dispositivo constituye un "bricolaje estratégico" que compagina la existencia de una agencia colectiva e impersonal con la ausencia de una estrategia de conjunto articulada conscientemente (2003: 52-53).

En resumen, el concepto es útil porque nos ayuda a pensar los fenómenos socio-culturales (Moro Abadía 2003: 40) y, en cierto sentido, a operacionalizarlos. Para ilustrar su relevancia para el desarrollo de mi tesis, retomaré las ideas centrales de una investigación fundamentada en este concepto, descubierta después de la estancia en Bolonia y cuyas analogías con mi problemática resultaron cruciales a la hora de adoptar esta perspectiva. Así pues, el estudio de Feldman (2011: 32-49) se ha realizado en la sede ginebrina del "Mediterranean Managed Migration Project" (3MP), una institución que junta a representantes de la Comisión Europea, la Organización para la Seguridad y la Cooperación en Europa, países africanos y expertos de varios tipos, con el objetivo de debatir y alcanzar acuerdos comunes sobre diferentes cuestiones relacionadas con la inmigración -control de las fronteras, datos biométricos, migraciones laborales, cuestiones demográficas, etc.-. El autor sostiene que el conjunto de los instrumentos predispuestos por la UE para armonizar la gestión de las migraciones, al interconectar diferentes niveles y dominios políticos (como los de seguridad, desarrollo, empleo, derechos humanos, etc.) y moldear un marco de comprensión compartido de ciertos fenómenos, puede ser considerado un dispositivo. También plantea que su etnografía puede definirse "no local", ya que, antes que implantarse en un único lugar, aboga por rastrear las conexiones de la misma problematización en diferentes espacios. En efecto, aunque ésta se ubique en un espacio geográfico específico (la sede del 3MP), en cierto sentido lo transciende, para centrarse en la conversación multisituada y transescalar que tiene lugar indirectamente entre los actores implicados. Este debate se da a través de diferentes espacios y mediante una pluralidad de formatos declaraciones públicas, producción de legislaciones nacionales, conferencias internacionales, folletos, sitios Internet, etc. Así, pues, el 3MP constituye una localización, pero no la única, desde la que es posible observar un proceso de entrelazamiento de discursos que se encauzan hacia una problematización común de las migraciones; discursos que están presentes en diferentes lugares sin ser exclusivos de ninguno en particular (Feldman 2011: 33).

Mediante esta última lectura llegué a entender que un conjunto de cosas tan dispares, como los diferentes instrumentos desplegados por el Marco Europeo de la Integración -un entrecruce de discursos, saberes, prácticas participativas, encarnados en diferentes instrumentos y mecanismos de coordinación y puestos en relación a través de una pluralidad de espacios y actores- , al estar atravesado por una problematización común de la integración y un esfuerzo de alineación gubernamental, podía ser analizado como un dispositivo. Si en un principio las relaciones inherentes a mi temática de estudio se habían presentado desconectadas -como si los diferentes instrumentos representaran "mundos aparte" (Marcus 1995: 102)-, por fin ahora captaba con claridad la interconexión existente entre las herramientas. A pesar de las diferencias, éstas estaban estructuradas por una relación saber/poder muy parecida y todas originaban de una visión (aparentemente) "despolitizada" y "tecnicista" de la integración. Por ejemplo, tanto las prácticas participativas del Foro, como el intercambio de las buenas prácticas y el proceso de construcción de los módulos aspiraban a producir un debate "no politizado" sobre la integración, alejado de los "sensacionalismos" y orientado a determinar las políticas "que funcionan" desde una perspectiva lo más "objetiva" posible. Esta concepción cientifista remitía claramente a unos rasgos comunes mediante los cuales la construcción social de la integración se estaba realizando.

A raíz de estas consideraciones, volví a modificar mi formulación de las unidades de análisis para darle su versión definitiva. Ahora ya seguía ocupándome del Foro Europeo sobre la Integración -del que, mientras tanto, había observado dos sesiones enteras-, pero sin darle una importancia central, ni tampoco entendiéndolo como el único lugar. Remitiendo a esa concepción relacional del campo, éste era sólo uno de los espacios de vertebración del "Marco Europeo de la Integración" y mi tesis se ocuparía del conjunto de los elementos de este dispositivo. Después del infructuoso intento por acotar de antemano el contexto de análisis, por fin, al dejarme llevar por el rastro de los actores y de las políticas públicas, había encontrado la 
manera de recorrer las articulaciones del campo y tantear su consistencia. Finalmente lograba enlazar espacios diferentes atravesados por una misma problematización. Desde este punto de vista Bruselas, al igual que Ginebra en el caso del 3MP, representaba una de las posibles localizaciones, sin duda muy relevante, desde la que mirar hacia el dispositivo, pero el dispositivo en su totalidad no era bajo ningún concepto "contenido" por Bruselas. Situarse en esa localización o en otra podía constituir una elección premeditada o una circunstancia posteriormente convertida en estrategia (como en mi caso), pero no dejaba de representar una perspectiva inevitablemente parcial, la cual resaltaría algunas cosas y ofuscaría otras.

\subsection{Consecuencias del nuevo enfoque}

La nueva conceptualización del contexto de estudio, madurada en una fase tardía y a raíz de intuiciones empíricas anteriores, conllevaría a su vez otra tanda de ajustes en la arquitectura definitiva del diseño de investigación. Quiero hacer hincapié en dos consecuencias particularmente interesantes:

1) A raíz del cambio de enfoque, ahora ya el estudio de las prácticas relacionadas con la integración de inmigrantes no estaba enfocado a analizar los universos culturales relevantes para los elaboradores de las políticas, sino a desentrañar los mecanismos de articulación del dispositivo por aquéllos definido. Por mucho que proporcionaran útiles informaciones de contexto, sus mundos sociales de procedencia ya no ocupaban un espacio central, siendo el objetivo de la investigación el análisis de aquéllos producidos por sus prácticas políticas (Shore y Wright 2011: 1). Así pues, mi objetivo no era el de centrarme en el homo communautarius (Abélès 2009) sin más, ni tampoco el de estudiar detalladamente la experiencia social relevante para el funcionariado de la Comisión (véase Shore 2000) o las ONG europeas. Más bien, se trataba de estudiar cómo estos actores, junto a otros, iban construyendo el dispositivo desde una localización específica; de qué manera iban definiendo sus fronteras; qué tipos de significados iban asociando a las medidas implementadas. Por lo tanto, el conocimiento del contexto de producción de aquellas prácticas seguía siendo una información útil y deseable, pero no constituía la finalidad principal de la investigación (6).

Aclararé la anterior afirmación con el siguiente ejemplo: numerosas etnografías sobre la UE han analizado los mecanismos mediante los cuales la interacción entre ciertos actores, en el contexto socio-cultural bruselense, ha producido significados políticos. Algunas han analizado la jerga inherente a la praxis burocrática cotidiana de la Comisión (véase Shore 2000); otras han mostrado la creciente difusión del Euroenglish, lengua franca asociada a la movilidad de las jóvenes generaciones de la clase media intelectual (Favell 2008); otras, finalmente, han ilustrado los procedimientos de traducción entre los diferentes idiomas en el Parlamento Europeo, resaltando la generación de un lenguaje institucional "gris" y "empobrecido", el Eurospeak (Abélès 2009). En cambio, el estatuto del lenguaje en mi investigación ha sido diferente. No he desentrañado los mecanismos de producción de significados compartidos, sino más bien me he centrado en cómo aquellos significados, aquellas formas peculiares de nombrar ciertos "objetos" han tenido efectos performativos a la hora de construir, (re)producir y (re)presentar una realidad social. Así pues, el estudio del proceso mediante el cual se ha constituido una forma específica de hablar sobre migración e integración ha ocupado un lugar secundario. Más bien, he privilegiado el análisis de las maneras en que esta construcción ha influido en la articulación de las políticas de integración comunitarias; en la definición e implementación de sus instrumentos; en la vertebración de prácticas políticas concretas. Por ejemplo, en lugar de centrarme en el origen de las distinciones entre "ciudadanos europeos" y "nacionales de terceros países" o entre "residentes legales" e "inmigrantes ilegales" (7), he desentrañado las consecuencias de esta operación. En efecto, he puesto de relieve que este marco discursivo hegemónico ha permitido legitimar la exclusión de ciertas categorías poblacionales del alcance del Fondo Europeo para la Integración (ciudadanos europeos y migrantes en condiciones "irregulares"); igualmente, ha dificultado la participación en el Foro de algunas organizaciones relacionadas con migrantes indocumentados. Especialmente en este último caso, he argumentado que este uso del lenguaje ha permitido la vertebración de procesos de clasificación de poblaciones y de construcción de la diferencia, contribuyendo a naturalizar las desigualdades existentes y a ocultar los mecanismos políticos y jurídicos vigentes de producción de la "irregularidad". Más allá de este ejemplo específico, el objetivo principal de mi estudio, impulsado por el cambio de enfoque, ha sido el de estudiar qué problematizaciones se iban produciendo a partir de las prácticas políticas y las configuraciones socio-culturales existentes en las diferentes articulaciones del dispositivo. Lo cual no quita la utilidad contextual de conocer también su proceso de constitución.

2) El estatuto de la observación participante se encontraba entonces profundamente modificado. En efecto, 
a la largo de mi trayectoria etnográfica la susodicha práctica se había visto claramente desbordada, a raíz de su incapacidad para abarcar un conjunto de dominios políticos y localizaciones tan amplio y transescalar como el del dispositivo estudiado. Mi enfoque se había valido de todo tipo de herramienta (aparte de las entrevistas): folletos, libros, leyes, declaraciones, recortes de periódicos, carteles, sitios web, mítines políticos, etc. Lo que es más importante, la recopilación de estos materiales se había dado en diferentes niveles y localizaciones, bajo ningún concepto reducibles al lugar "Bruselas" ni tampoco únicamente a un espacio físico que se pudiera observar de manera continuada. La selección de los materiales relevantes no había estado orientada por el alcance de un muestreo exhaustivo y holístico de "todo lo existente", sino por la búsqueda de la representatividad cualitativa de las relaciones existentes. La práctica etnográfica por mí realizada, aun sin excluir el recurso a la observación participante, me había llevado a re-contextualizar profundamente su importancia, además de acompañarla de otras estrategias investigadoras, a menudo decididas sobre la marcha a raíz de su eficacia en un momento dado. Estas formas de acercamiento a la realidad eran en cierto sentido más "conceptuales" y menos "directas" (Feldman 2011: 47), en la medida en que no se fundamentaban (solamente) en la experiencia de la observación, sino que también se atrevían a establecer conexiones entre fenómenos multisituados y de diversos tipos.

Por otra parte, en una fase tardía descubriría que algunas lecturas ya recomendaban fuertemente la práctica de cierto pluralismo metodológico y de las fuentes. Por ejemplo, Gupta y Ferguson critican el arquetipo malinowskiano del trabajo de campo, según el cual es posible adquirir conocimiento certero sólo mediante la observación directa y un contacto estrecho y cotidiano con las poblaciones observadas (1997: 15). Este planteamiento, indudablemente eficaz en muchos sentidos, conlleva la exclusión o la desvalorización de todas aquellas formas de producción de conocimiento menos "extrañas" y exóticas, originadas de relaciones menos localizadas y que no pueden agotarse dentro de unas coordenadas geográficas estables y limitadas -sin por eso llegar a ser menos antropológicas- (Gupta y Ferguson 1997: 15). De ahí que algunos planteen la necesidad de "descentrar" la idea tradicional de campo, alejarse del paradigma de las etnografías "realistas" y hasta llegar a "indisciplinar los procedimientos en la producción del saber con nuevas experimentaciones" (García y otros 2011: 207). Como es evidente, las anteriores aportaciones me resultaban atractivas, ya que describían eficazmente la estrategia etnográfica que había estado desarrollando y me permitían fundamentarla teóricamente.

Para concluir esta sección: al igual que el objeto de estudio definitivo de una tesis guarda poco parecido con el inicial, también el contexto se modifica a lo largo de la trayectoria investigadora. En mi caso, los "dóndes" han pasado de delimitar lugares meramente geográficos a indicar espacios (independientemente de que fueran "físicos" o "virtuales") atravesados por relaciones transescalares. La definición de los límites del campo, lejos de ser una fase previa abstractamente ajena a la experiencia de la "entrada" material en él, se ha dado mediante una aproximación continua y en gran parte empírica. Para que se disipara la indeterminación inicial había sido necesario "escuchar" al campo (Arribas 2011: 788), recorrerlo, ir "tanteando" sus confines para manejar las dimensiones de análisis adecuadas. lgualmente, los actores principales se han ido "revelando" sobre la marcha: conforme iba "rastreando" las políticas públicas relevantes, ellas me indicaban quiénes eran los "nativos" implicados en su elaboración/implementación/evaluación. Las relaciones habían de ser detectadas en el entramado de las políticas públicas y no en las especificidades de un lugar.

Una vez más, llegué a dar coherencia teórica a la investigación en una fase muy "tardía", cuando ya gran parte del trabajo se había hecho. En este sentido, la misma elaboración de este artículo teóricometodológico puede considerarse como un producto del proceso investigador antes que una premisa de su desarrollo.

\section{Conclusiones}

Este artículo constituye un ejemplo más de una argumentación sostenida por numerosos estudiosos: y es que la investigación científica, especialmente (aunque no sólo) en las ciencias sociales, dista mucho de corresponderse a ese patrón "logicista" y "escolástico" en el que la producción de conocimiento es afectada únicamente por factores teóricos y metodológicos "internos" a las disciplinas (Bourdieu 2003: 73). Por el contrario, el procedimiento investigador es un recorrido no-lineal, "precario" (Arribas 2011: 788) y a veces incierto, marcado por ajustes continuos e influido por las vivencias, las disposiciones y los condicionantes socio-culturales, económicos y logísticos de quienes investigan. En el caso de mi tesis, estos 
constreñimientos han influido de diferentes maneras; por ejemplo, a la hora de no poder "volver atrás" todas las veces que me topé con iluminaciones teóricas relevantes, teniendo más bien que re-adaptar y rectificar sobre la marcha lo que había elaborado hasta la fecha y sacándole todo el jugo posible a entrevistas que no podría volver a realizar. En este sentido, el recorrido investigador ha sido una verdadera "travesía", se ha nutrido de condicionantes de todo tipo y se ha ido afinando sobre la marcha, ajustándose a exigencias nuevas y anteriormente no pensadas, re-enfocándose a cada paso. La caja de herramientas ha sido continuamente puesta a prueba.

Por lo que respecta a los contenidos específicos de mi proceso investigador, éste ha estado marcado por dos grandes desplazamentos, relativos al objeto y el contexto de estudio, cuya consecuencia principal ha sido un relevante cambio de enfoque. Por lo que respecta al objeto, había empezado la tesis con una perspectiva (al menos parcialmente) cientifista, politológica y poco deconstructiva, para terminar realizando un análisis crítico de las relaciones de saber/poder hegemónicas. Mediante la antropología de las políticas públicas pude entender la categoría de integración como un producto histórico y socio-cultural, mientras que a través de los estudios sobre gubernamentalidad llegué a conceptualizarla como una herramienta para el gobierno de algunas poblaciones migrantes, inserta en una dinámica de "despolitización" y "tecnificación" de ciertas políticas públicas y vertebradora de procesos de inclusión/exclusión. En otras palabras: estas aproximaciones me invitaban a centrarme en la performatividad de las prácticas políticas analizadas, en su capacidad para producir realidad social. En cuanto al contexto, había llegado a Bruselas con una idea "geográfica" de campo en la cabeza, para entender más tarde que, aunque esa localización encajara bien dentro de mi diseño, lo haría en un sentido diferente al concebido inicialmente. No se trataba de ir a la "capital de Europa", sino de ubicarse, desde la perspectiva de la etnografía "no local" (Feldman 2011), en un "nodo" privilegiado que me permitiera recorrer el entramado relacional y transescalar de un dispositivo político. Lo cual conllevaba, entre otras cosas, relativizar la importancia de la observación participante, recontextualizándola como una de las prácticas posibles dentro de un abanico más amplio de estrategias etnográficas, no todas necesariamente fundamentadas en la experiencia "directa" y "sensible". Puedo decir a posteriori que se ha tratado de dos desplazamientos muy interesantes, que me han permitido ver cosas anteriormente borrosas; al igual que, por supuesto, habrán ofuscado otras, dado que la perspectiva finalmente adoptada no tiene porqué constituir el único acercamiento posible, ni tampoco necesariamente el mejor.

\section{Notas}

1. De la que el presente texto es parte integrante. Su realización ha sido financiada por el Programa $6 \mathrm{~A}$ del Plan Propio de Investigación de la Universidad de Granada. Las citas directas de textos en inglés son traducciones propias.

2. La diferenciación entre "objeto" y "contexto" presente en el título de los apartados 2 y 3 responde sobre todo a exigencias de organización de la información. Eso no quita que los dos elementos, al estar caracterizados por una relación de profunda interdependencia, aparezcan juntos en algunas reflexiones. Además, soy consciente de que también existe una dimensión temporal (un "cuándo"), que sin embargo no será tratada en el presente texto.

3. Desde luego, es posible que su papel en el futuro llegue a ser más relevante. La perspectiva metodológica aquí propuesta tiene en cuenta la naturaleza dinámica de las relaciones analizadas.

4. Esta arquitectura institucional se fundamenta en la idea de "gobernanza multinivel", difundida a partir de finales de los años noventa dentro del paradigma neoliberal del new public management. Al remitir a mecanismos más "abiertos", "flexibles" y "horizontales" de elaboración de las políticas, esta filosofía no simplemente aspira a determinar una alineación de las conductas entre los diferentes actores, sino también a optimizar el rendimiento de los mismos mecanismos decisionales y a reducir sus gastos. Analizada desde la perspectiva de la gubernamentalidad, expresa una racionalidad que ha sido llamada "gobierno a distancia" (Rose y Miller 1992: 9) o "gobierno aún más a distancia” (Walters y Haahr 2005: 118).

5. Esta concepción relacional del contexto de investigación presenta fuertes analogías con la noción bourdieusiana de campo (véase Bourdieu 2003 y Bourdieu y Wacquant 2005): de hecho, en mi posterior 
estancia en Bolonia leí algunos textos de sociología constructivista de la Unión Europea fundamentados en los conceptos desarrollados por el francés (véanse Guiraudon 2000, 2003 y 2011, Favell y Guiraudon 2009, Kauppi 2011 y Mérand, 2011), los cuales me proporcionarían ulteriores herramientas para contextualizar la emergencia de las políticas europeas de integración y entender su proceso de constitución dentro de un campo político determinado.

6. Este procedimiento también presentaba claras analogías con el trabajo de Feldman. En su muy poco exótico estudio de personas trabajando en una institución, la mayoría del tiempo sentadas frente a ordenadores y rodeadas de aparatos tecnológicos, el objetivo del trabajo de campo no había sido el de aprehender los mundos sociales relevantes para aquéllos, ni desentrañar el sentido de sus prácticas cotidianas. Más bien se había tratado de analizar de qué manera, a partir de los susodichos mundos y prácticas, los actores iban reforzando un marco común de comprensión y armonización de ciertas políticas, delimitando la gama de las alternativas posibles y pensables (Feldman 2011: 35-37).

7. Es menester señalar que esta última expresión ha sido recientemente reemplazada en el lenguaje oficial por la de "irregulares", de acuerdo con las recomendaciones de la actual Comisaria de Interior Cecilia Malmström.

\section{Bibliografía}

Abélès, Marc

2000 "Virtual Europe", en Irene Bellier y Thomas M. Wilson (eds.), An anthropology of the European Union. Oxford-New York, Berg: 31-52.

2009 "The Practitioners of Europe", en Riva Kastoryano (ed.), An Identity for Europe. The Relevance of Multiculturalism in EU Construction. New York, Palgrave Macmillan: 27-44.

Abélés, Marc (y otros)

1993 An Anthropological Approach to the European Commission. Brussels, European Commission.

Agrela, Belén

2006 Análisis antropológico de las políticas sociales dirigidas a la población inmigrante. Tesis Doctoral. Granada, Departamento de Trabajo Social y Servicios Sociales.

Arribas, Alberto

2011 "Contradicción, contingencia, y deseo. Micropolítica de (mi) investigación", en A.A.V.V., Libro de Actas del XII Congreso de Antropología de la F.A.A.E.E.. León, Universidad de León: 787-791.

Bauman, Zygmunt

2003 "De peregrino a turista, o una breve historia de la identidad", en Stuart Hall y Paul Du Gay (coords.), Cuestiones de identidad cultural. Buenos Aires, Amorrortu: 40-68.

Bellier, Irène

1997 "The Commission as an Actor: an Anthropologist's View", en Helen Wallace y Alasdair Young (eds.), Participation and Policy-making in the European Union. Oxford, Clarendon Press.

2000 "The European Union, Identity Politics and the Logic of Interests' Representation", en Irène Bellier y Thomas M. Wilson (eds.), An anthropology of the European Union. Oxford-New York, Berg: 53-73.

Bellier, Irène (y Thomas M. Wilson)

2000 "Building, Imagining and Experiencing Europe: Institutions and Identities in the European Union", en Irène Bellier y Thomas M. Wilson (eds.), An anthropology of the European Union, Oxford-New York, Berg: 127.

Bhaba, Homi K.

2003 "El entre-medio de la cultura", en Stuart Hall y Paul Du Gay (coords.), Cuestiones de identidad cultural. Buenos Aires, Amorrortu: 94-106.

Bourdieu, Pierre

2003 El oficio de científico. Ciencia de la ciencia y reflexividad. Barcelona, Anagrama. 
Bourdieu, Pierre (y Loïc Wacquant)

2005 Una invitación a la sociología reflexiva. Madrid, Siglo XXI.

Burchell, Graham (y otros) (eds.)

1991 The Foucault Effect. Studies in governmentality. With two lectures by and an interview with Michel Foucault". Chicago, University of Chicago Press.

Castel, Robert

1997 Las metamorfosis de la cuestión social: una crónica del salariado. Buenos Aires, Paidós.

Cruikshank, Barbara

1999 The will to empower. Democratic citizens and other subjects. Ithaca-London, Cornell University Press.

De Lucas, Javier

2003 "La inmigración, como res política", en AA.VV., Movimientos de personas e ideas y multiculturalidad (vol. II). Bilbao, Universidad de Deusto: 193-225.

Dean, Mitchell

2010 Governmentality. Power and Rule in a Modern Society. London, Sage.

Favell, Adrian

2000 Philoshopy of integration: immigration and the idea of citizenship in France and Britain. Houndmills Basingstoke, Macmillan.

2006 "The nation-centered perspective", en Marco Giugni y Florence Passy, Dialogues on Migration Policy. Lanham, MD, Lexington: 45-56.

2008 Eurostars and Eurocities. Free movement and mobility in an integrating Europe. Malden (MA), Blackwell.

Favell, Adrian (y Viriginie Guiraudon)

2009 "The Sociology of the European Union: An Agenda", European Union Politics, n 10: 550-576.

Feldman, Gregory

2011 "Illuminating the Apparatus: Steps toward a Nonlocal Ethnography of Global Governance", en Cris Shore (y otros) (eds.), Policy worlds. Anthropology and the Analysis of Contemporary Power. New YorkOxford, Berghahn Books: 32-49.

Feyerabend, Paul

1987 Contra el método. Esquema de una teoría anarquista del conocimiento. Barcelona, Ariel.

Foucault, Michel

1985 Saber y verdad. Madrid, La Piqueta.

1991 "Governmentality", en Graham Burchell (y otros) (eds.), The Foucault Effect. Studies in governmentality. With two lectures by and an interview with Michel Foucault. Chicago, University of Chicago Press: 87-104.

2009 Las palabras y las cosas: una arqueología de las ciencias humanas. Madrid, Siglo XXI.

García, Francisco Javier (y otros)

2011 "Prismas transescalares en el estudio de las migraciones", Revista de Antropología Social, $\mathrm{n}^{\circ} 20$ : 203-228.

Gil, Sandra

2006 Las argucias de la integración. Tesis doctoral. Madrid, Universidad Complutense de Madrid.

Guiraudon, Virginie

2000 "European Integration and Migration Policy: Vertical Policy-making as Venue Shopping", Journal of Common Market Studies, $n^{\circ}$ 38(2): 251-71.

2003 "The constitution of a European immigration policy domain: a political sociology approach", Journal of European Public Policy, ${ }^{\circ}$ 10(2): 263-282.

2011 "Mobilization, Social Movements and the Media", en Adrian Favell y Virginie Guiraudon (eds.), 
Sociology of the European Union. Basingstoke, Palgrave Macmillan: 128-149.

Gupta, Akhil (y James Ferguson)

1997 "Discipline and Practice: 'The Field' as Site, Method, and Location in Anthropology", en Akhil Gupta y James Ferguson (eds.), Anthropological locations: boundaries and grounds of a field science. Berkeley/Los Angeles, University of California Press: 1-46.

Hall, Stuart

2003 “Introducción: ¿quién necesita identidad?", en Stuart Hall y Paul Du Gay (coords.), Cuestiones de identidad cultural. Buenos Aires, Amorrortu: 13-39.

Haraway, Donna

1995 Ciencia, cyborgs y mujeres. La reinvención de la naturaleza. Madrid, Cátedra.

Hesse, Mary

1980 Revolutions and reconstructions in the philosophy of science. Brighton, Harvester Press.

Kauppi, Niilo

2011 "EU Politics", en Adrian Favell y Virginie Guiraudon (eds.), Sociology of the European Union. Basingstoke, Palgrave Macmillan: 150-171.

Kuhn, Thomas

1975 The Structure of Scientific Revolutions. Chicago, University of Chicago.

Latour, Bruno

2005 Re-Assembling the Social. An Introduction to Actor-Network Theory. Oxford, Oxford University Press.

Marcus, George

1995 "Ethnography in/of the World System: the Emergence of Multi-Sited Ethnography", Annual Review of Anthropology, $\mathrm{n}^{\circ}$ 24: 95-117.

Mérand, Frédéric

2011 "EU policies", en Adrian Favell y Virginie Guiraudon (eds.), Sociology of the European Union. Basingstoke, Palgrave Macmillan: 172-192.

Mignolo, Walter

2009 "Epistemic Disobedience, Independent Thought and De-Colonial Freedom", Theory, Culture \& Society, $\mathrm{n}^{\circ} 26(7-8): 1-23$.

Morin, Edgar

2004 "La epistemologia de la complejidad", Gazeta de Antropología, n 20.

http://www.gazeta-antropologia.es/?p=2841.

Moro Abadía, Óscar

2003 “¿Qué es un dispositivo?”, Empiria. Revista de Metodología de Ciencias Sociales, n 6: 29-46.

Rabinow, Paul

2003 Anthropos Today: reflections on modern equipment. Princeton, NJ, Princeton University Press.

Rose, Nikolas

1999 Powers of freedom. Reframing political thought. Cambridge, Cambridge University Press.

Rose, Nikolas (y Peter Miller)

1992 "Political power beyond the State: problematics of government", British Journal of Sociology, $\mathrm{n}^{\circ} 43$ (2): 173-205.

Sayad, Abdelmalek

2002 La doppia assenza. Dalle illusioni dell'emigrato alle sofferenze dellïmmigrato. Milano, Cortina Editore. 
Shore, Cris

1993 “Inventing the 'People's Europe': Critical Perspectives on European Community Cultural Policy", Man, n $\circ$ 28: 779-800.

2000 Building Europe: The Cultural Politics of European Integration. London, Routledge.

2001 European Union and the Politics of Culture, Occasional Paper, $n^{\circ}$ 43. London, Bruges Group.

2010 "La antropología y el estudio de la política pública: reflexiones sobre la 'formulación' de las políticas", Antipoda, $\mathrm{n}^{\circ}$ 10: 21-49.

Shore, Cris (y Annabel Black)

1996 "Citizens' Europe and the Construction of European Identity", en Victoria A. Goddard (y otros), The Anthropology of Europe. Identities and boundaries in conflict. Oxford, Berg: 275-298.

Shore, Cris (y Susan Wright)

1997 Anthropology of Policy. Critical perspectives on Governance and power. London, Routledge.

2011 "Conceptualising Policy: Technologies of Governance and the Politics of Visibility", en Cris Shore (y otros) (eds.), Policy worlds. Anthropology and the Analysis of Contemporary Power. Nueva York-Oxford, Berghahn Books: 1-25.

Shore, Cris (y otros) (eds.)

2011 Policy worlds. Anthropology and the Analysis of Contemporary Power. Nueva York-Oxford, Berghahn Books.

Walters, Williams (y Jens H. Haahr)

2005 Governing Europe. Discourse, governmentality and european integration. London/New York, Routledge.

Wimmer, Andreas (y Nina Glick Schiller)

2002 "Methodological nationalism and beyond: nation-state building, migration and the social sciences", Global Networks, n² 2( 4): 301-334.

Wright, Susan

2011 "Studying policy: methods, paradigms, perspectives", en Cris Shore (y otros) (eds.), Policy worlds. Anthropology and the Analysis of Contemporary Power. Nueva York-Oxford, Berghahn Books: 27-31. 